\title{
Impact of Smartphone Notification Display Choice in a Typing Task
}

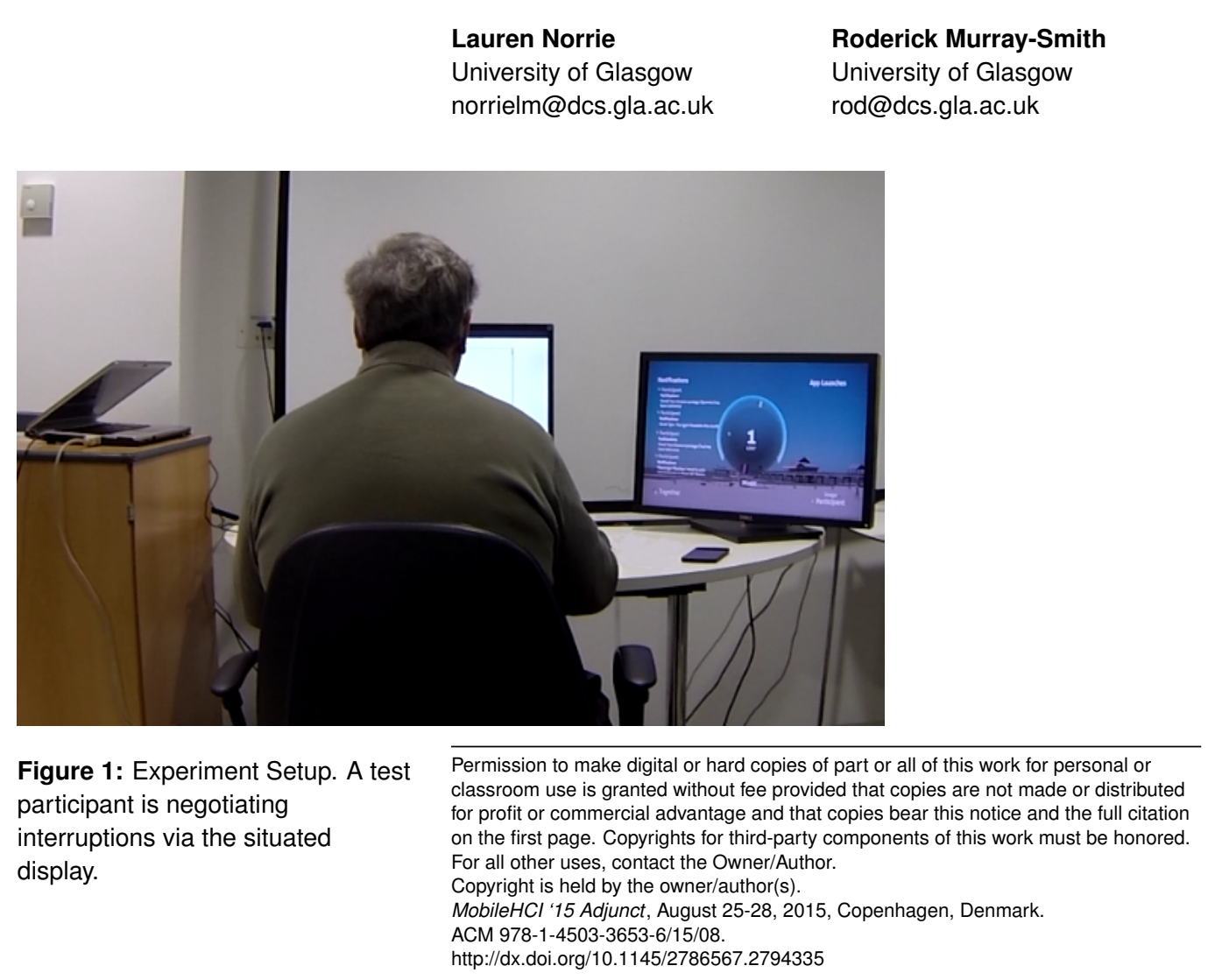

\begin{abstract}
External displays have the potential to make smartphone notifications less obtrusive when a user has committed their attention to a primary task. We compare six notification displays, and evaluate the impact that negotiating smartphone interruptions has on a typing task when the number of notifications to ignore and act on are equal. A lab experiment with 30 participants is conducted, and initial results show that desktop pop-ups are preferred significantly more, where they require the fewest actions to read. Managing notifications via the notification bar is least preferred, despite requiring fewer actions to respond. This work is a well-controlled pre-cursor to the application of notification displays in social scenarios. The results motivate the use of external displays to manage attention around smartphone interruptions.
\end{abstract}

\section{Author Keywords}

Smartphone, Desktop, Typing, Notifications.

\section{ACM Classification Keywords}

H.5.2 [Information interfaces and presentation]: UI

\section{Introduction}

Smartphone notifications provide awareness of important emails and messages. However, without consideration of the user and their context, notifications can be distracting, and frequent interruptions can result in stress. Negotiated interruptions [3] let the user to decide the onset of an interruption, and is an approach to managing notifications that can improve concentration on a primary task. 


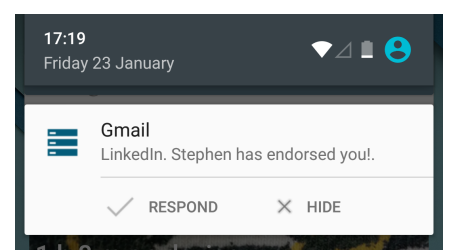

(a) NB (Android 5.0)

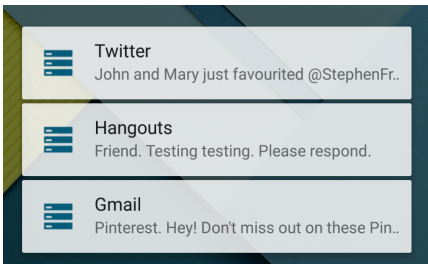

(b) LS (Android 5.0)

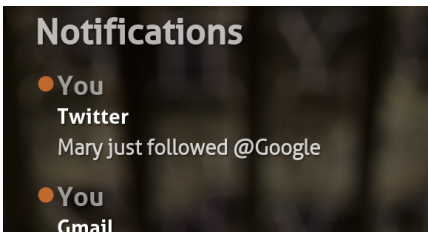

(c) SD (Cast Together)

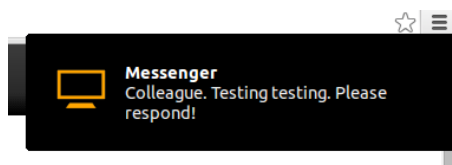

(d) DP (Linconnect)

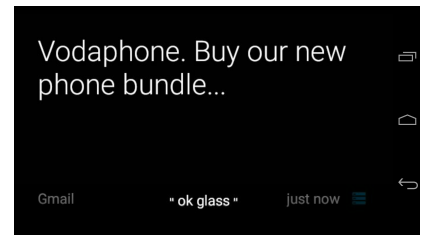

(e) SE (Google Glass)

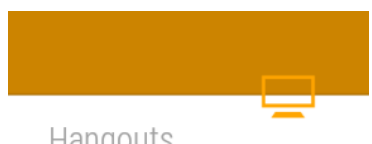

Boss. Are you around after the meeting?

Please respond!

(f) SW (Moto 360)

Figure 2: Notification displays.
On an Android smartphone, users negotiate interruptions via the notification bar (NB). The NB displays a list of recent notifications in a pull-down menu, and is always one step away when the device is in use. However, when the smartphone is not in use, the NB can require many actions to read the notification. Displaying notifications on the lockscreen (LS) can reduce the cost of reading a notification when the device is in hand but not unlocked. Compared to the NB, the cost of responding to the notification from the LS is increased, as the device has still to be unlocked. However, by reading the notification at a glance, prolonged smartphone diversion could be less likely when attention is committed to a typing task, or a co-located social situation.

External displays create new opportunities to deliver notifications to the user. Smartwatches (SW) and smart eyewear (SE) allow users to read notifications when the device is not in hand, by looking towards the wrist or glancing upwards. Notifications can also be displayed on a monitor as desktop pop-ups (DP), or on a situated display in the user environment $(S D)$. The choice of an external notification display has the potential to increase focus on a task by making it easier to read notifications, but more difficult to respond. As new ways of reading smartphone notifications become available, it is important to consider the impact that they will have on attention to everyday tasks.

This work contributes a study of six smartphone notification displays, shown in Figure 2, and their impact on attention to a typing task. Though many notifications do not require a response or can be ignored [5, 4], we select an equal number to be ignored and acted on to compare the relative importance of each notification type on subjective opinion.

\section{User Experiment}

30 participants took part in a 1.5 hour lab experiment, and were asked to manage notifications while performing six 10 minute typing tasks. A $£ 10$ reward was provided for participation. The experiment setup can be seen in Figure 1.

\section{Participants}

Participants were aged 18 - 35 (mean=25, st.dev. $=5$ ), 10 were female. 26/30 participants studied Computing Science and were recruited from an undergraduate mailing list.

A preliminary questionnaire was provided to understand any source of bias. On a 7-point Likert scale, all participants reported to type on a keyboard regularly $(6.63+/-0.82)$, and were more likely to receive smartphone notifications $(6.28$ $+/-0.91)$ than to respond $(5.25+/-1.30)$. Participants were most likely to position the smartphone beside them (left or right) while typing at a computer (27/30), which is in line with the design of the experiment. Allowing participants to choose the onset of an interruption fits with the design of negotiated interruptions: $16 / 30$ participants would finish a task before checking notifications, and 12/30 participants would allow notifications to interrupt their task. Few participants would wait until a predefined time of day. Participants were asked which display they would choose to read smartphone notifications while typing at a desktop PC. 16/30 participants indicated that they would choose a smartphone display, and 9/30 chose a DP or SD, and only 4/30 chose a wearable.

\section{Typing Task}

A custom text editor was implemented as a web page to log key strokes as participants typed phrases during the typing task. Phrases were consistently displayed in a single line at the top of the page to minimise head movements during the typing task. The next phrase appeared when a newline was entered in the editor.

We merged two popular phrase sets for text entry $[2,6]$ to create a large collection of simple phrases. Phrases were localised for UK English and phrases with names and numbers were removed. The remaining phrases were randomised 


\section{Participant Quotes}

Notification bar: 'This method [is used] in my everyday life and I'm tired of it' [P20].

Lockscreen: 'It was faster than using the basic notification bar for non-urgent notifications' [P11].

Situated display: 'I could glance at the display to check the notification without my hands leaving the keyboard' [P14].

Desktop pop-up: 'Everything's all in the one place. You only need to look away from the screen if the message is urgent' [P7].

Smart eyewear: 'Only I could see the notifications. Better privacy than when notifications are displayed on your laptop' [P15].

Smartwatch: 'Less distracting than I thought it would be. It was easy to glance at the screen and understand the notification without moving too much' [P9].
Lockscreen. With the LS, participants 'liked the fact that I could see the notification without unlocking the phone' [P10] and 'it was faster than using the basic notification bar for non-urgent notifications' [P11], but disliked that 'I still had to turn on my display to read it, distracting me from whatever I was doing' [P20] and 'you still had to take your hands off the keyboard' [P27]. The LS could be used 'pretty much anywhere, even though it might be quite obvious you're checking your phone' [P22] and 'when I need a glance overview of notifications (check periodically without unlocking phone)' [P8]. Several participants reported that ' $\mathrm{do}$ use this as my default, I suppose. But I don't exactly like it' [P9] and would use the LS 'only where no other option was available' [P6].

Situated display. Participants 'liked that [the SD] didn't interrupt what I was doing so much and felt it was easier to concentrate... It was less urgent to pick up the smartphone as I knew what the notification was about already' [P13]. Several participants reported that 'I could glance at the display to check the notification without my hands leaving the keyboard' [P14]. Participants felt that it was 'easy to concentrate on writing at the same time' [P22]. However, participants disliked that 'the background changed... I sometimes thought that something was trying to get my attention over there when it maybe wasn't' [P9] and 'because the display was very big, it was hard to read the messages and therefore took some time to read it and was easily distracted.' [P18]. Participants 'would definitely use it either when I am working at home or at the office since I receive notifications regularly' [P20].

Desktop pop-up. The DP made it 'very convenient to look at the notification because it's literally on the screen I'm already looking at' [P24] and 'everything's all in the one place. You only need to look away from the screen if the message is urgent' [P7]. Several participants thought that 'it's a bit disturbing when you are working, as notifications will frequently pop up on the same screen' [P5]. P13 'felt that I had to respond more urgently than the prior experiment [SD] as previously it was to the side of me and somehow I could almost prioritise more easily and finish my typing where as when it was on the monitor I felt I had to do it right then'. DP could be used in a 'daily working situation' [P5], and some participants 'use something similar already (Pushbullet)' [P27].

Smart Eyewear. Participants liked 'not having to move my hands from the keyboard' [P11], and 'only I could see the notifications. Better privacy than when they are displayed on your laptop' [P15]. Participants did not like 'the narrow field of view' [P3], 'having to move my head up to trigger the display' [P11] and 'the notification did not appear immediately on the Glass screen' [P26]. P24 found the 'head gesture feel very unnatural and awkward, gesture detection was inconsistent leading me to view the notification on the phone instead'. Participants could imagine using SE while 'doing a task that involved more complicated use of your hands than typing, like if you were wearing gloves or something that would make it harder to check on your phone' [P11], 'when outside walking about, not necessarily when inside using a desktop computer' [P7] and 'carrying out a task where I needed to pay attention to it, but where there wasn't already a screen in front of me (cooking, walking)' [P9].

Smartwatch. Participants liked that the SW 'was outside your vision so wasn't a distraction. The vibration was very short - let you know something was there but left you alone quickly' [P8]. P1 liked that 'I tend to look at my hands while typing, so it was easy to glance down to my wrist (easier than other methods)'. The SW was 'less distracting than I thought it would be. It was easy to glance at the screen and understand the notification without moving too much' [P9]. Several participants felt 'it was annoying that you had to lift your hand to read the notification. This meant stopping typing. It was also quite heavy on the wrist, so it was a bit an- 
Overall Preference

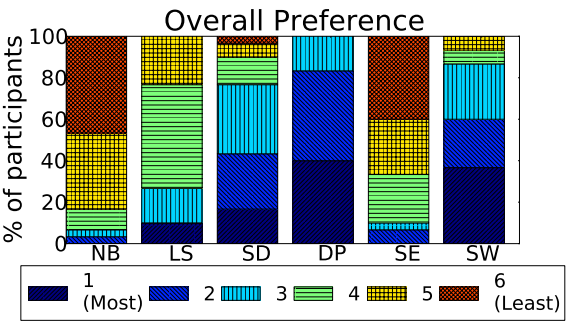

Figure 5: Overall preference. Desktop Pop-ups (DP) are most preferred, and the notification bar (NB) is least preferred.

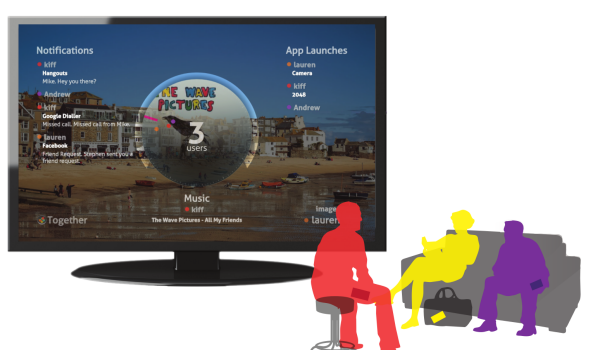

Figure 6: Cast Together is a situated display (SD) prototype designed to support multiple smartphone users in an intimate co-located situation, who can negotiate interruptions on a shared display without removing the smartphone from a bag or pocket.

\section{Acknowledgements}

We are grateful to EPSRC for Ph.D. funding. noying to type with' [P14]. Participants could imagine using the SW 'while hanging out with friends I would prefer using a fast method to check my notifications like this, rather than having to take out the phone of my pocket' [P10], 'receiving notifications in public when wanting to be discreet' [P1] and 'if I was out and didn't want to constantly check my phone in my pocket' [P14].

\section{Overall Preference}

The ranking of each display is displayed in Figure 5. The DP was preferred most overall (12/30). In final comments, participants shared that 'pop-ups were the best cause it was the quickest way to decide whether the notification was important or not' [P15], 'the desktop pop-up required least effort to read' [P14] and 'really liked the desktop and situated display as it was easy to interpret' [P30]. Many participants (10/30) preferred the SW most. In the final comments, P3 shared that the watch was my favourite, with its bright notification screen it was handy for seeing what needed my attention and did not interrupt with the task', and P7 stated 'need to get me a smart watch'. The preference of SW was surprising, as few participants expected to choose a wearable in the preliminary questionnaire. In addition to the concerns over the comfort of wearing a SW while typing, visual appeal was also considered to be important. P15 felt that the watch was great as well [but] the notifications were not that well displayed on it though'. The NB was least preferred, despite the majority of participants choosing smartphone displays in the preliminary questionnaire. Interestingly, the NB was not preferred significantly less than SE $(P=0.169)$. Participants felt that '[SE] required the most [effort to read]' [P14], and 'apart from the Google Glass device, all notification methods seemed quite easy to read without disrupting my current task very often' [P26]. P9 acknowledged that 'some of the notification displays would have ranked differently had I not been using a computer at the time'. Figure 6 highlights the application of our SD as a way for multiple smartphone users to negotiate interruptions in a social context.

\section{Discussion}

We presented our initial results from a comparison of six smartphone notification displays in a typing task, where notifications required a fast response or could be ignored. External displays were rated significantly higher than smartphone displays overall, despite requiring more actions to respond to a notification. The data collected will allow more detailed analysis of typing performance and resumption lag. This work is a controlled pre-cursor to the application of notification displays in social scenarios. Future work should consider the impact of notification display choice in a social setting with personal devices and notifications.

\section{References}

[1] Andrés Lucero and Akos Vetek. 2014. NotifEye: Using Interactive Glasses to Deal with Notifications While Walking in Public (ACE '14). 17:1-17:10.

[2] I. Scott MacKenzie and R. William Soukoreff. 2003. Phrase Sets for Evaluating Text Entry Techniques ( $\mathrm{CH}$ EA '03). 754-755.

[3] Daniel McFarlane. 2002. Comparison of Four Primary Methods for Coordinating the Interruption of People in Human-computer Interaction. Hum.-Comput. Interact. (2002), 63-139.

[4] Martin Pielot, Karen Church, and Rodrigo de Oliveira. 2014. An In-situ Study of Mobile Phone Notifications (MobileHCl '14). 233-242.

[5] Alireza Sahami Shirazi, Niels Henze, Tilman Dingler, Martin Pielot, Dominik Weber, and Albrecht Schmidt. 2014. Large-scale Assessment of Mobile Notifications (CHI '14). 3055-3064.

[6] Keith Vertanen and Per Ola Kristensson. 2011. A Versatile Dataset for Text Entry Evaluations Based on Genuine Mobile Emails (MobileHCl '11). 295-298. 